\title{
HARMONIC DIFFEOMORPHISMS BETWEEN HADAMARD MANIFOLDS
}

\author{
PETER LI, LUEN-FAI TAM AND JIAPING WANG
}

\begin{abstract}
In this paper, we study the Dirichlet problem at infinity for harmonic maps between complete hyperbolic Hadamard surfaces. We will address the existence and uniqueness questions relating to the problem. In particular, we generalize results in the work of Li-Tam and Wan.
\end{abstract}

\section{INTRODUCTION}

In a series of papers [L-T 1], [L-T 2] and [L-T 3], the first two authors considered the existence, uniqueness and boundary regularity theory for the Dirichlet problem at infinity for proper harmonic maps between hyperbolic spaces. If we denote $\mathbb{H}^{m}$ to be the $m$-dimensional hyperbolic space with constant -1 sectional curvature, then using the Poincare disk model, we can represent $\mathbb{H}^{m}$ by the unit $m$-disk $\mathbb{D}^{m}$ endowed with the Poincaré metric $d s_{\mathbb{H}^{m}}^{2}=\frac{4}{\left(1-\rho^{2}\right)^{2}} d s_{0}^{2}$, where $\rho$ denotes the Euclidean distance to the origin and $d s_{0}^{2}$ is the Euclidean metric. The geometric compactification is given by the natural compactification of $\mathbb{D}^{m}$ by the unit $(m-1)$-sphere $\mathbb{S}^{m-1}$. Using this identification, a map from $\mathbb{S}^{m-1}$ to $\mathbb{S}^{n-1}$ can be viewed as a map from infinity of $\mathbb{H}^{m}$ to infinity of $\mathbb{H}^{n}$. Let us summarize some of the results in [L-T 1], [L-T 2] and [L-T 3] as follows:

Theorem (Li-Tam). Let $\phi: \mathbb{S}^{(m-1)} \rightarrow \mathbb{S}^{(n-1)}$ be a $C^{1}$ map which has nowhere vanishing energy density. Then there exists a unique harmonic extension $h$ : $\mathbb{H}^{m} \rightarrow \mathbb{H}^{n}$ of $\phi$ which is in $C^{1}\left(\overline{\mathbb{D}}^{m}, \overline{\mathbb{D}}^{n}\right)$. Also, if $\phi$ is in $C^{k, \alpha}\left(\mathbb{S}^{(m-1)}, \mathbb{S}^{(n-1)}\right)$, for some $1 \leq k \leq m$ and some $0<\alpha$, then $h$ is in $C^{k, \gamma}\left(\overline{\mathbb{D}}^{m}, \overline{\mathbb{D}}^{n}\right)$ for some $0<\gamma<\alpha$.

We would like to mention that the existence part of this theorem for the special case when $m=2=n$ and for $C^{3}$ boundary maps which have nowhere vanishing energy density was independently proved by Akutagawa [A].

Using a different point of view, Wan [W] studied harmonic diffeomorphisms between hyperbolic 2-spaces via their associated Hopf differentials. By using the fact that [C-T] the Gauss map of a constant mean curvature cut in a Minkowski space-time is a harmonic map into hyperbolic space, Wan established that there is a one-to-one correspondence between the set of equivalent classes of constant mean curvature cuts and the equivalent classes of quadratic differentials in $\mathbb{H}^{2}$.

Received by the editors December 12, 1994.

1991 Mathematics Subject Classification. Primary 58E20.

The authors were partially supported by NSF grant \#DMS9300422.

(c) 1995 American Mathematical Society 
The equivalent relation on the set of constant mean curvature cuts is given by the isometries and the equivalent relation on the set of quadratic differentials is given by composition with Möbius transformations. In the second part of his paper, Wan also proved that:

Theorem (Wan). Let $h: \mathbb{H}^{2} \rightarrow \mathbb{H}^{2}$ be an orientation preserving harmonic diffeomorphism of hyperbolic 2-spaces. Then $h$ is quasi-conformal if and only if $h$ has uniformly bounded energy density.

In this paper, the authors will, to some extent, generalize the above theorems to the class of hyperbolic Hadamard surfaces which are complete, simply connected, Riemannian surfaces with Gaussian curvature $K$ bounded by $-\kappa \leq K \leq 0$ for some constant $0<\kappa$ and have positive lower bounds for their spectra. In the first section, we will show that a hyperbolic Hadamard surface is quasi-isometrically and conformally equivalent to the hyperbolic 2-space $\mathbb{H}^{2}$ with constant curvature -1 . Part of the argument (Theorem 1.1) which involves estimating the energy density of a conformal map from $\mathbb{H}^{2}$ into a hyperbolic Hadamard surface can be generalized to higher dimension and is interesting in its own right.

In $\S 2$, we will show that (Corollary 2.1) if $h: M \rightarrow N$ is a harmonic diffeomorphism between two hyperbolic Hadamard surfaces, then $h$ has uniformly bounded energy density if and only if $h$ is quasi-conformal. This generalizes Wan's theorem to this category of surfaces.

In the point of view of solving the Dirichlet problem at infinity, we will consider the existence theory in $\S 3$. Since a hyperbolic Hadamard surface is quasi-isometrically and conformally equivalent to $\mathbb{H}^{2}$, we can identify its geometric boundary with $\mathbb{S}^{1}$. Let $M$ and $N$ be two hyperbolic Hadamard surfaces. For some $0<\alpha$, let $\phi: \mathbb{S}^{1} \rightarrow \mathbb{S}^{1}$ be a $C^{1, \alpha}$ map which has nowhere vanishing energy density, then we will show that (Corollary 3.1) $\phi$ has a harmonic extension $h: M \rightarrow N$ which is $C^{1, \gamma}$ up to the boundary for any $0<\gamma<\alpha$. Note that we have not been able to prove the existence theorem for $C^{1}$ boundary maps. This is due to the fact that we are not assuming that the target manifold has strictly negative curvature.

Finally, in $\S 4$, we will prove a uniqueness theorem similar to the above stated theorem of Li-Tam. We will show that if $h_{1}, h_{2}: M \rightarrow N$ are two harmonic maps between two hyperbolic Hadamard surfaces which are $C^{1}$ up to the boundary and share the same boundary map $\phi$ that has nowhere vanishing energy density, then $h_{1} \equiv h_{2}$.

The authors would like to thank Tom Wan for many valuable discussions and his interest in this work.

\section{QUASI-CONFORMALITY IMPLIES BOUNDED ENERGY}

Theorem 1.1. Let $M$ be a complete $n$-dimensional manifold with Ricci curvature bounded from below by $-(n-1) \kappa_{M}$ for some constant $\kappa_{M} \geq 0$. Suppose $N$ is an $n$-dimensional manifold with non-positive sectional curvature and that the lower bound of the spectrum of $N$ is positive and bounded by $\lambda>0$. If $h: M \rightarrow N$ is a quasi-conformal harmonic diffeomorphism from $M$ onto its image, then there exists a constant $C\left(n, \kappa_{M}, \lambda, \mu\right)>0$ depending only on $n, \kappa_{M}, \lambda$, and the quasi-conformal constant $\mu$, such that $e(h) \leq C$. 
Proof. The fact that $\lambda>0$ is the lower bound of the spectrum of $N$ implies that

$$
\int_{N}\left|\nabla_{N} f\right|^{2} d V_{N} \geq \lambda \int_{N} f^{2} d V_{N}
$$

for all smooth functions $f$ with compact support on $N$. Let $g$ be a smooth function with compact support. Suppose $n>2$, for any $\alpha>1$, let $f=g^{\alpha}$ and apply Schwarz inequality, we have

$$
\begin{aligned}
& \alpha^{2}\left(\int_{N}|g|^{\frac{(2 \alpha-2) n}{n-2}} d V_{N}\right)^{\frac{n-2}{n}}\left(\int_{N}\left|\nabla_{N} g\right|^{n} d V_{N}\right)^{\frac{2}{n}} \\
& \quad \geq \alpha^{2} \int_{N}|g|^{2 \alpha-2}\left|\nabla_{N} g\right|^{2} d V_{N} \\
& \quad=\int_{N}\left|\nabla_{N} g^{\alpha}\right|^{2} d V_{N} \\
& \quad \geq \lambda \int_{N}|g|^{2 \alpha} d V_{N} .
\end{aligned}
$$

By choosing $\alpha=\frac{n}{2}$, this implies that

$$
\int_{N}\left|\nabla_{N} g\right|^{n} d V_{N} \geq\left(\frac{2 \sqrt{\lambda}}{n}\right)^{n} \int_{N}|g|^{n} d V_{N}
$$

The above inequality is obviously true for $n=2$. Using the fact that $h$ is a diffeomorphism, the inequality can be written as

$$
\int_{N}\left|\nabla_{N} \phi \circ h^{-1}\right|^{n} d V_{N} \geq\left(\frac{2 \sqrt{\lambda}}{n}\right)^{n} \int_{N}\left|\phi \circ h^{-1}\right|^{n} d V_{N}
$$

for all smooth functions $\phi$ with compact support on $M$. The chain rule and the identity $d\left(h^{-1}\right)=(d h)^{-1}$ assert that if

$$
a(x)=\inf _{\left\{v \in M_{x}|| v \mid=1\right\}}|d h(v)|
$$

then

$$
\left|\nabla_{N}\left(\phi \circ h^{-1}\right)\right|(x) \leq a^{-1}(x)\left|\nabla_{M} \phi\right|(x) .
$$

Observe that the Jacobian $J(h)$ of $h$ is given by $d V_{N}=J(h) d V_{M}$ and

$$
a(x)^{n} \leq J(h)(x) \leq\left(\frac{e(h)(x)}{n}\right)^{\frac{n}{2}} .
$$

On the other hand, quasi-conformality implies that

$$
e(h)(x) \leq\left(1+(n-1) \mu^{2}\right) a(x)^{2} .
$$

Hence

$$
\left(\frac{e(h)(x)}{\left(1+(n-1) \mu^{2}\right)}\right)^{\frac{n}{2}} \leq J(h)(x) \leq\left(\frac{\left(1+(n-1) \mu^{2}\right) a(x)^{2}}{n}\right)^{\frac{n}{2}} .
$$

We conclude that

$$
\int_{M}\left|\nabla_{M} \phi\right|^{n} d V_{M} \geq\left(\frac{2 \sqrt{\lambda}}{\sqrt{n}\left(1+(n-1) \mu^{2}\right)}\right)^{n} \int_{M}|\phi|^{n} e(h)^{\frac{n}{2}} d V_{M}
$$


For a fixed point $p \in M$, let us choose $\phi$ to be a smooth function supported on $B_{p}(2)$ with the property that

(1) $\phi=1$ on $B_{p}(1)$,

(2) $\phi \geq 0$ on $M$, and

(3) $\left|\nabla_{M} \phi\right| \leq 3$ on $M$.

Applying (1.1) to $\phi$, we have

$$
3^{n} V_{x}(2) \geq\left(\frac{2 \sqrt{\lambda}}{\sqrt{n}\left(1+(n-1) \mu^{2}\right)}\right)^{n} \int_{B_{x}(1)} e(h)^{\frac{n}{2}} d V_{M}
$$

On the other hand, since $M$ has Ricci curvature bounded from below by $-(n-1) \kappa_{M}$, the volume comparison theorem implies that $V_{x}(2) \leq$ $C\left(n, \kappa_{M}\right) V_{x}(1)$, for some constant depending only on $n$ and $\kappa_{M}$. Hence there exists a constant $C_{1}\left(n, \kappa_{M}, \lambda, \mu\right)>0$ such that

$$
C_{1} \geq V_{x}(1)^{-1} \int_{B_{x}(1)} e(h)^{\frac{n}{2}}
$$

Since $h$ is a harmonic map, the Bochner formula for the energy density gives the inequality

$$
\Delta e(h) \geq-(n-1) \kappa_{M} e(h) .
$$

Applying the mean value inequality as obtained in [L-T 1], we conclude that there exists a constant $C_{2}\left(n, \kappa_{M}, \lambda, \mu\right)>0$ such that

$$
C_{2} \geq e(h)(p)
$$

for all $p \in M$. Hence the theorem is proved.

Corollary 1.1. Let $M$ be a complete surface with metric given by $d s_{M}^{2}$ and has Gaussian curvature bounded from below by $-\kappa_{M}$ for some constant $\kappa_{M} \geq 0$. Suppose $N$ is another surface with metric given by $d s_{N}^{2}$ which has non-positive curvature and a positive lower bound $\lambda$ for the spectrum. If $M$ and $N$ are conformally equivalent given by a conformal map $h: M \rightarrow N$, such that

$$
h^{*}\left(d s_{N}^{2}\right)=\psi d s_{M}^{2}
$$

for some positive function $\psi$. Then there exists a constant $C\left(\kappa_{M}, \lambda\right)>0$ depending only on $\kappa_{M}$ and $\lambda$ such that $\psi \leq C\left(\kappa_{M}, \lambda\right)$.

Proof. The corollary follows from Theorem 1, the facts that a conformal map is harmonic in dimension 2 and the energy of $h$ is given by $\psi$.

Corollary 1.2. Let $M$ be a complete surface which has non-positive Gaussian curvature and is also bounded from below by $-\kappa_{M}$ for some $\kappa_{M}>0$. Suppose the universal covering $\tilde{M}$ of $M$ has a positive lower bound for the spectrum, then there exists a complete surface $N$ with constant -1 curvature that is quasiisometrically and conformally equivalent to $M$. Moreover, any two hyperbolic Hadamard surfaces must be conformally and quasi-isometrically equivalent to each other.

Proof. The uniformization theorem asserts that $M$ is conformally equivalent to a complete surface with constant -1 curvature. Let $h: M \rightarrow N$ be the conformal map. Consider $\tilde{h}: \tilde{M} \rightarrow \mathbb{H}^{2}$ to be the lift of $h$ to the universal coverings of $M$ and $N$. We can write

$$
\tilde{h}^{*}\left(d s_{\mathbb{H}^{2}}^{2}\right)=\psi d s_{\tilde{M}}^{2} .
$$


Applying Corollary 1.1 to $\tilde{h}^{-1}: \mathbb{H}^{2} \rightarrow \tilde{M}$, we conclude that $\psi^{-1}$ is bounded from above. On the other hand, a generalized Schwarz lemma of Yau [Y] asserts that $\psi \leq \kappa_{M}$. Hence $\psi$ is uniformly bounded away from 0 and $\infty$. This proves the first part of the Corollary. When $M$ is simply connected, $N$ can be taken to be the hyperbolic space and the second part of the Corollary follows immediately.

\section{BOUNDED ENERGY IMPLIES QUASI-CONFORMALITY}

Theorem 2.1. Let $M$ be a hyperbolic Hadamard surface, and $N$ be a complete simply connected surface with Gaussian curvature $K_{N}$ satisfying

$$
0 \geq K_{N} \geq-\kappa_{N}
$$

for some constant $\kappa_{N}>0$. Suppose $h: M \rightarrow N$ is a harmonic diffeomorphism from $M$ onto $N$. Then $h$ is quasi-conformal if its energy density $e(h)$ is uniformly bounded.

Proof. Let us first observe that in dimension 2, harmonicity is a conformal invariant for the domain manifold. On the other hand, by the uniformization theorem, $M$ is conformally equivalent to the hyperbolic space. If we write the hyperbolic metric as $d \bar{s}^{2}=\psi d s_{M}^{2}$, then the energy density $\bar{e}(h)$ of $h$ with respect to the hyperbolic metric is given by $\bar{e}(h)=\psi^{-1} e(h)$. By Corollary 1.2, $\psi$ is uniformly bounded away from 0 and $\infty$. Therefore it suffices to prove the theorem for $M$ being the hyperbolic space.

In this case, Wan's argument [W] with some slight modifications will provide the proof. We will give an outline here for the sake of completeness. Using the same notation as in [W], in terms of local holomorphic coordinates, we have

$$
\begin{aligned}
\Delta \log |\partial h| & =-K_{N}\left(|\partial h|^{2}-|\bar{\partial} h|^{2}\right)-1 \\
& =-K_{N}|\partial h|^{2}\left(1-\mu^{2}\right)-1
\end{aligned}
$$

where $\mu=\frac{|\bar{\partial} h|}{|\partial h|}$ is the local quasi-conformal constant. The fact that $h$ is a diffeomorphism implies that

$$
J(h)=|\partial h|^{2}-|\bar{\partial} h|^{2}>0,
$$

hence

$$
\mu<1 \text {. }
$$

To establish the fact that $h$ is quasi-conformal, we need to prove that $\mu \leq 1-\epsilon$ for some $\epsilon>0$. If we write $w=\log |\partial h|$, then (2.1) becomes

$$
e^{-2 w} \Delta(-w)=K_{N}\left(1-\mu^{2}\right)+e^{-2 w} \text {. }
$$

Let us define a new metric by $d \tilde{s}^{2}=|\partial h|^{2} d s_{M}^{2}$. Since $h$ is a diffeomorphism (see page 10 of [S]), the pull-back metric $h^{*}\left(d s_{N}^{2}\right)$ is dominated from above by $2 d \tilde{s}^{2}$, hence $d \tilde{s}^{2}$ is also a complete metric. In terms of the Laplacian of the metric $d \tilde{s}^{2}$, equation (2.2) can be written as

$$
\tilde{\Delta}(-w)=K_{N}\left(1-\mu^{2}\right)+e^{-2 w} .
$$

The generalized maximum principle of $[\mathrm{C}-\mathrm{Y}]$ implies that $-w$ is bounded from above with the upper bound given by

$$
\kappa_{N} \geq e^{-2 w}
$$


Therefore

$$
|\partial h|^{2} \geq \frac{1}{\kappa_{N}} .
$$

The assumption that $h$ has bounded energy density and the identity

$$
e(h)=|\partial h|^{2}+|\bar{\partial} h|^{2}
$$

implies that $|\partial h|^{2}$ is also bounded from above. It follows that $d \tilde{s}^{2}$ is uniformly equivalent to $d s_{M}^{2}$. For any given $\alpha>0$, there exists $r_{0}>0$ such that the volume of geodesic balls for the metric $d s_{M}^{2}$ satisfies

$$
V_{x}(r) \geq \exp (\alpha r)
$$

for any $r \geq r_{0}$ and $x \in M$. Hence there exists $r_{1}>0$ such that this estimate on the volume is also valid for the metric $d \tilde{s}^{2}$ for all $r \geq r_{1}$ and for all $x \in M$. However, (2.1) implies that the Gaussian curvature for the metric $d \tilde{s}^{2}$ is given by

$$
\tilde{K}=K_{N}\left(1-\mu^{2}\right)
$$

which is non-positive and bounded from below by $-\kappa_{N}$. Hence, the simply connected manifold $M$ together with the metric $d \tilde{s}^{2}$ has no cut-points. GaussBonnet asserts that

$$
\int_{\tilde{B}_{x}(r)} \tilde{K}=-\int_{\partial \tilde{B}_{x}(r)} \tilde{k}_{g}+2 \pi
$$

where $\tilde{k}_{g}$ is the geodesic curvature with respect to the unit outward normal vector and $\tilde{B}(r)$ is the geodesic ball centered at $x$ with radius $r$ for the metric $d \tilde{s}^{2}$. However, the fact that $d \tilde{s}^{2}$ has no cut-points implies that

$$
\int_{\partial \tilde{B}_{x}(r)} \tilde{k}_{g}=\frac{\partial^{2} \tilde{V}_{x}(r)}{\partial r^{2}}
$$

Hence, together with (2.4), we conclude that

$$
\tilde{V}_{x}(r)=\int_{0}^{r} \int_{0}^{t}\left(2 \pi-\int_{\tilde{B}_{x}(r)} \tilde{K}\right) .
$$

The exponential estimate of $\tilde{V}_{x}(r)$ implies that

$$
\exp (\alpha r) \leq \int_{0}^{r} \int_{0}^{t}\left(2 \pi-\int_{\tilde{B}_{x}(r)} \tilde{K}\right)
$$

for $r \geq r_{1}$. The non-positivity of $\tilde{K}$ imples that for a fixed sufficiently large $r$, we have

$$
\int_{\tilde{B}_{x}(r)} \tilde{K} \leq-\kappa_{N}
$$

for all $x$. Together with (2.3) this implies that

$$
\int_{\tilde{B}_{x}(r)}\left(1-\mu^{2}\right) \geq 1
$$

On the other hand, a direct computation as in [W] shows that

$$
\widetilde{\Delta} \log \mu=K_{N}|\partial h|^{2}\left(1-\mu^{2}\right)
$$


where $\tilde{\Delta}$ is the Laplacian on $M$ with respect to the metric $d \tilde{s}^{2}$. Hence, in terms of the metric $d \tilde{s}^{2}$ we can write this as

$$
\widetilde{\Delta}\left(1-\mu^{2}\right) \leq-2 K_{N}\left(1-\mu^{2}\right) \leq 2 \kappa_{N}\left(1-\mu^{2}\right) .
$$

Since $d \tilde{s}^{2}$ is quasi-isometric to the hyperbolic space, the de-Gorgi-Nash-Moser theory asserts that there is a constant $C>0$ such that

$$
1-\mu^{2}(x) \geq C \int_{\tilde{B}_{x}(r)}\left(1-\mu^{2}\right)
$$

Applying (2.5), we conclude that $\mu$ is uniformly bounded away from 1 , and that $h$ is quasi-conformal.

Combining Theorems 1.1 and 2.1 and lifting the harmonic map to the universal coverings, we obtain the following generalization of Wan's theorem.

Corollary 2.1. Let $M$ and $N$ are surfaces whose universal coverings $\tilde{M}$ and $\tilde{N}$ are hyperbolic Hadamard surfaces. Let $h: M \rightarrow N$ be a harmonic diffeomorphism from $M$ onto its image which induces an injection of $\pi_{1}(M)$ into $\pi_{1}(N)$. Then $h$ is quasi-conformal if and only if its energy density is uniformly bounded.

\section{EXISTENCE THEORY}

Let $d s^{2}$ and $d \bar{s}^{2}=e^{2 u} d s^{2}$ be two conformally equivalent metrics defined on an $n$-dimensional Riemannian manifold $N$. Suppose $M$ is an $m$-dimensional Riemannian manifold and $h: M \rightarrow N$ is a $C^{2}$ mapping from $M$ into $N$. Let $\left\{\theta_{i}\right\}_{1 \leq i \leq m}$ be orthonormal coframes of the manifold $M$. The first structural equations are given by

$$
d \theta_{i}=\theta_{i j} \wedge \theta_{j}
$$

with

$$
\theta_{i j}=-\theta_{j i} \text {. }
$$

Similarly, let $\left\{\omega_{\alpha}\right\}_{1 \leq \alpha \leq n}$ be orthonormal coframes of the manifold $N$ with respect to the metric $d s^{2}$, and $\left\{\bar{\omega}_{\alpha}\right\}_{1 \leq \alpha \leq n}$ be orthonormal coframes with respect to the metric $d \bar{s}^{2}$. Since $d \bar{s}^{2}=e^{2 u} d s^{2}$, the coframes can be taken to be

$$
\bar{\omega}_{\alpha}=e^{u} \omega_{\alpha} .
$$

Their first structural equations are given by

$$
d \omega_{\alpha}=\omega_{\alpha \beta} \wedge \omega_{\beta}, \quad \omega_{\alpha \beta}=-\omega_{\beta \alpha}
$$

and

$$
d \bar{\omega}_{\alpha}=\bar{\omega}_{\alpha \beta} \wedge \bar{\omega}_{\beta}, \quad \bar{\omega}_{\alpha \beta}=-\bar{\omega}_{\beta \alpha} .
$$

Direct computation yields that

$$
\bar{\omega}_{\alpha \beta}=\omega_{\alpha \beta}+u_{\alpha} \omega_{\beta}-u_{\beta} \omega_{\alpha}
$$

For a $C^{2}$ map $h: M \rightarrow N$, the energy densities of $h$ with respect to the two metrics $d s^{2}$ and $d \bar{s}^{2}$ are given by

$$
e(h)=\sum_{\alpha, i}\left(a_{\alpha i}\right)^{2}
$$


and

$$
\bar{e}(h)=\sum_{\alpha, i}\left(\bar{a}_{\alpha i}\right)^{2}
$$

where $a_{\alpha i}$ and $\bar{a}_{\alpha i}$ are defined by

$$
h^{*}\left(\omega_{\alpha}\right)=a_{\alpha i} \theta_{i}
$$

and

$$
h^{*}\left(\bar{\omega}_{\alpha}\right)=\bar{a}_{\alpha i} \theta_{i}
$$

By (3.1), we conclude that

$$
\bar{a}_{\alpha i}=e^{u \circ h} a_{\alpha i} .
$$

The tension field of $h$ with respect to the metric $d s^{2}$ in terms of the orthonormal coframes $\left\{\omega_{\alpha}\right\}$ is given by

$$
\tau_{\alpha}=\sum_{i} a_{\alpha i i}
$$

where $a_{\alpha i i}$ is determined by the expression

$$
\sum_{j} a_{\alpha i j} \theta_{j}=d a_{\alpha i}-\sum_{j} a_{\alpha j} \theta_{i j}+\sum_{\beta} a_{\beta i} h^{*}\left(\omega_{\beta \alpha}\right) .
$$

Similarly, the tension field of $h$ with respect to the metric $d \bar{s}^{2}$ in terms of the orthonormal coframes $\left\{\bar{\omega}_{\alpha}\right\}$ is given by

$$
\bar{\tau}_{\alpha}=\sum_{i} \bar{a}_{\alpha i i}
$$

with

$$
\sum_{j} \bar{a}_{\alpha i j} \theta_{j}=d \bar{a}_{\alpha i}-\sum_{j} \bar{a}_{\alpha j} \theta_{i j}+\sum_{\beta} \bar{a}_{\beta i} h^{*}\left(\bar{\omega}_{\beta \alpha}\right) .
$$

Exterior differentiating (3.3) yields

$$
d \bar{a}_{\alpha i}=e^{u \circ h} d a_{\alpha i}+e^{u \circ h}\left(u_{\beta} \circ h\right) a_{\beta j} a_{\alpha i} \theta_{j} .
$$

Substituting this, (3.2) and (3.3) into (3.4) gives

$$
\begin{aligned}
\sum_{j} \bar{a}_{\alpha i j} \theta_{j}=e^{u \circ h} & \sum_{j} a_{\alpha i j} \theta_{j} \\
& +e^{u \circ h}\left(\left(u_{\beta} \circ h\right) a_{\beta j} a_{\alpha i}+\left(u_{\beta} \circ h\right) a_{\beta i} a_{\alpha j}-\left(u_{\alpha} \circ h\right) a_{\beta i} a_{\beta j}\right) \theta_{j} .
\end{aligned}
$$

Hence the tension fields are related by

$$
\bar{\tau}_{\alpha}=e^{u \circ h}\left(\tau_{\alpha}+2 \sum_{\beta, i}\left(u_{\beta} \circ h\right) a_{\beta i} a_{\alpha i}-\left(u_{\alpha} \circ h\right) \sum_{\beta, i}\left(a_{\beta i}\right)^{2}\right) .
$$

Let us now consider the case when $N$ is a complete simply connected surface with Gaussian curvature satisfying $-\kappa_{N} \leq K_{N} \leq 0$ for some constant $\kappa_{N}>0$. We assume that $N$ is given by the unit disk $\mathbb{D}^{2}$ in $\mathbb{R}^{2}$ with metric $d s^{2}=$ $e^{2 u} d s_{\mathbf{H}^{2}}^{2}$, where $d s_{\mathbb{H}^{2}}^{2}$ is the hyperbolic metric, and that $u$ is bounded. Let $\varphi$ be a conformal map which maps the upper half-space of $\mathbb{R}^{2}$ to $\mathbb{D}^{2}$, then $N$ can also be identified as the upper half-space with metric $e^{2 u \circ \varphi} d s_{\mathbf{H}^{2}}^{2}$. If we use 
the Poincare disk model for $N$, so that the metric of $N$ is $d s^{2}=\frac{4 e^{2 u}}{\left(1-r^{2}\right)^{2}} d s_{0}^{2}$, where $d s_{0}^{2}$ is the Euclidean metric on $\mathbb{D}^{2}$, and $r$ is the Euclidean distance to the origin, then

$$
\Delta_{N} u=-1-K_{N} e^{2 u} .
$$

By the assumption that both $u$ and $K_{N}$ are bounded and using Corollary 1.2, we have

$$
\Delta_{0} u=O\left((1-r)^{-2}\right),
$$

and by Theorem 3.9 in [G-T], this implies that

$$
\left|\nabla_{0} u\right|=O\left((1-r)^{-1}\right) \text {, }
$$

where $\Delta_{0}$ is the Euclidean Laplacian and $\nabla_{0}$ is the Euclidean gradient. We are now ready to prove an existence theorem for the Dirichlet problem at infinity from $\mathbb{H}^{2}$ to $N$.

Theorem 3.1. Let $\mathbb{H}^{2}$ be the standard 2-dimensional hyperbolic space. Let $N$ be a complete surface such that $N=\left(\mathbb{D}^{2}, d s^{2}\right)$, where $d s^{2}=e^{2 u} d s_{\mathbb{H}^{2}}^{2}$, and $d s_{\mathrm{H}^{2}}^{2}$ is the hyperbolic metric. Suppose $u$ is uniformly bounded in $\mathbb{D}^{2}$, and the Gaussian curvature of $N$ satisfies the bound $-\kappa_{N} \leq K_{N} \leq 0$ for some constant $\kappa_{N}>0$. Let $\phi: \mathbb{S}^{1} \rightarrow \mathbb{S}^{1}$ be a $C^{1, \alpha}$ map from the standard circle to itself which has nowhere vanishing energy density for some $0<\alpha \leq 1$. If the geometric boundary of $\mathbb{H}^{2}$ at infinity with respect to the hyperbolic metric is identified with $\mathbb{S}^{1}$, then there exists a harmonic map $h: \mathbb{H}^{2} \rightarrow N$ such that $h=\phi$ on $\mathbb{S}^{1}$. Furthermore, if $h$ is viewed as a map from $\overline{\mathbb{D}}^{2}$ to $\overline{\mathbb{D}}^{2}$, then $h$ is $C^{1, \gamma}$ up to the boundary for all $0<\gamma<\alpha$.

Proof. Theorem 4.2 of [L-T 2] asserts that there exists a solution $\tilde{h}$ to the Dirichlet problem for harmonic map from $\mathbb{H}^{2}$ into $\mathbb{H}^{2}$ such that $\tilde{h}=\phi$ on $\mathbb{S}^{1}$. Moreover, $\tilde{h}$ has bounded energy density with respect to the hyperbolic metric on $\mathbb{D}^{2}$ and hence also with respect to the metric $d s^{2}$ on $N$. In addition, $\tilde{h}$ is $C^{1, \gamma}$ up to the boundary for all $0<\gamma<\alpha$, when viewed as a map from $\overline{\mathbb{D}}^{2}$ to $\overline{\mathbb{D}}^{2}$. The formula (3.5) between the tension fields of $\tilde{h}$ with respect to the hyperbolic metric and the metric $d s^{2}$ on $\mathbb{D}^{2}$ implies that

$$
\bar{\tau}_{\alpha}(\tilde{h})=e^{u \circ \tilde{h}}\left(2 \sum_{\beta, i}\left(u_{\beta} \circ \tilde{h}\right) a_{\beta i} a_{\alpha i}-\left(u_{\alpha} \circ \tilde{h}\right) \sum_{\beta, i}\left(a_{\beta i}\right)^{2}\right),
$$

where $\alpha=1,2$, and $\bar{\tau}_{\alpha}$ is the component of the tension field of $\tilde{h}$ with respect to the metric $d s^{2}$ in $N$. However, by Lemma 1.3 of [L-T 2], $\tilde{h}$ must be conformal on the boundary $\mathbb{S}^{1}$, and if $\mathbb{H}^{2}$ is identified with $\mathbb{D}^{2}$ with metric $\frac{4}{\left(1-\rho^{2}\right)^{2}} d s_{0}^{2}$ where $d s_{0}^{2}$ is the Euclidean metric, then

$$
C^{-1} \leq \frac{1-\rho(p)}{1-r(\tilde{h}(p))} \leq C
$$

for some constant $C>0$. Since $\tilde{h}$ is $C^{1, \gamma}$, when computed in terms of the hyperbolic metric

$$
\left|a_{\beta i}-\delta_{\beta i}\right|=O\left((1-\rho)^{\gamma}\right)
$$


as $p$ tends to the geometric boundary of $\mathbb{H}^{2}$. By (3.5)-(3.9), we have

$$
\|\bar{\tau}(\tilde{h})\|=O\left((1-\rho)^{\gamma}\right)
$$

as $p \rightarrow \infty$. This implies that $\|\tau(h)\| \in L^{q}\left(\mathbb{H}^{2}\right)$ for sufficiently large $q$. By Theorem 5.2 of [L-T 1], $\tilde{h}$ can be deformed to a harmonic map $h: \mathbb{H}^{2} \rightarrow N$ which has $\phi$ as a boundary map. In order to prove that $h$ is $C^{1, \gamma}$ up to the boundary, note that by Theorem 5.2 of [L-T 1], $h$ has bounded energy density and $d_{N}(\tilde{h}(p), h(p)) \rightarrow 0$ as $p \rightarrow \infty$. Using the fact that $K_{N} \leq 0$, we may proceed as in the proof of Lemma 3.3 in [L-T 2] to obtain

$$
|\tilde{h}(p)-h(p)|=O\left((1-\rho(p))^{1+\gamma} \log \left(\frac{1}{1-\rho(p)}\right)\right)
$$

as $p \rightarrow \infty$. If we use the upper half-space models for $\mathbb{H}^{2}$ and $N$, and write $\tilde{h}(x, y)=(\tilde{f}(x, y), \tilde{g}(x, y))$ and $h(x, y)=(f(x, y), g(x, y))$ with $y>0$ and $g, \tilde{g}>0$, then

$$
\Delta_{0} f=u_{1}\left(\left|\nabla_{0} g\right|^{2}-\left|\nabla_{0} f\right|^{2}\right)-2\left(u_{2}-\frac{1}{g}\right)\left\langle\nabla_{0} f, \nabla_{0} g\right\rangle
$$

and

$$
\Delta_{0} g=-\left(u_{2}-\frac{1}{g}\right)\left(\left|\nabla_{0} g\right|^{2}-\left|\nabla_{0} f\right|^{2}\right)-2 u_{1}\left\langle\nabla_{0} f, \nabla_{0} g\right\rangle
$$

where $u_{1}=\frac{\partial u}{\partial f} \circ h$ and $u_{2}=\frac{\partial u}{\partial g} \circ h$. By (3.11), it is easy to see that $g(x, y)$ and $\tilde{g}(x, y)$ are comparable as $y \rightarrow 0$. Since $h$ has bounded energy density, so $f$ and $g$ have bounded Euclidean gradients near $y=0$ for bounded $x$. Using these facts and (3.11)-(3.13), one can proceed as in the proof of Theorem 4.2 in [L-T 2] and show that $h$ is $C^{1, \gamma^{\prime}}$ up to the boundary for all $0<\gamma^{\prime}<\gamma$. This completes the proof of the theorem.

Corollary 3.1. Let $M$ and $N$ be hyperbolic Hadamard surfaces. Then according to Corollary 1.2, $M$ and $N$ are quasi-isometrically and conformally equivalent to $\mathbb{H}^{2}$. Let $\phi: \mathbb{S}^{1} \rightarrow \mathbb{S}^{1}$ be a $C^{1, \alpha}$ map from the standard circle to itself which has nowhere vanishing energy density for some $0<\alpha \leq 1$. If the geometric boundaries of $M$ and $N$ at infinity are identified with $\mathbb{S}^{1}$, then there exists a harmonic map $h: M \rightarrow N$ such that $h=\phi$ on $\mathbb{S}^{1}$. Furthermore, if $h$ is viewed as a map from $\overline{\mathbb{D}}^{2}$ to $\overline{\mathbb{D}}^{2}$, then $h$ is $C^{1, \gamma}$ up to the boundary for all $0<\gamma<\alpha$.

\section{UNIQUENESS THEORY}

Let $N$ be a hyperbolic Hadamard surface. Then by Corollary 1.2, $N$ is given by the unit disk $\mathbb{D}^{2}$ in $\mathbb{R}^{2}$ with metric $d s^{2}=e^{2 u} d s_{\mathbb{H}^{2}}^{2}$, where $d s_{\mathbb{H}^{2}}^{2}$ is the hyperbolic metric. Suppose $\varphi$ is a conformal map which maps the upper half-space of $\mathbb{R}^{2}$ to $\mathbb{D}^{2}$, then $N$ can also be identified as the upper half-space with metric $e^{2 u \circ \varphi} d s_{\mathbb{H}^{2}}^{2}$.

Lemma 4.1. Let $q_{0}$ be a boundary point at infinity of $N$. Using the upper half-space model for $N$, we may assume $q_{0}=\left(f_{0}, 0\right)$. Let us denote $U$ to be the intersection of the Euclidean ball $B_{q_{0}}(r)$ with the upper half-space, for 
some $r>0$. Given an $\epsilon>0$ there is a constant $0<\beta<1$ such that for all $q=(f, g), \tilde{q}=(\tilde{f}, \tilde{g})$ in $U$ and $|q-\tilde{q}| \leq \beta g$, we have

$$
\left|g \nabla_{0} u(q)-\tilde{g} \nabla_{0} u(\tilde{q})\right| \leq \epsilon .
$$

Proof. As in the proof of Theorem 3.1, using the fact that $u$ is bounded and $\Delta_{0} u=O\left(g^{-2}\right)$ where $\Delta_{0}$ is the Euclidean Laplacian, Theorem 3.9 in [G-T] asserts that there are constants $C_{1}, C_{2}>0$ such that for all $q=(f, g)$ in $U$,

$$
\sup _{B_{q}\left(\frac{1}{2} g\right)}|u|+g \sup _{B_{q}\left(\frac{1}{2} g\right)}\left|\nabla_{0} u\right|+g^{2} \sup _{B_{q}\left(\frac{1}{2} g\right)}\left|\Delta_{0} u\right| \leq C_{1}
$$

and

$$
\begin{aligned}
& \frac{d_{q \tilde{q}}^{2}}{|q-\tilde{q}|}\left|\nabla_{0} u(q)-\tilde{g} \nabla_{0} u(\tilde{q})\right| \\
& \quad \leq C_{2}\left(\sup _{B_{q}\left(\frac{1}{2} g\right)}|u|+\sup _{B_{q}\left(\frac{1}{2} g\right)} d_{q}^{2}\left|\Delta_{0} u\right|\right)\left(\left|\log \frac{d_{q \tilde{q}}}{|q-\tilde{q}|}\right|+1\right),
\end{aligned}
$$

where $d_{q \tilde{q}}=\min \left(d_{q}, d_{\tilde{q}}\right)$, and $d_{q^{\prime}}$ is the Euclidean distance of a point $q^{\prime}$ to $\partial B_{q}\left(\frac{1}{2} g\right)$. Here and in what follows, the notation $B_{q}(t)$ denotes the Euclidean ball of radius $t$ with center at $q$. Let $q$ and $\tilde{q}$ as in the lemma, then

$$
\left|g \nabla_{0} u(q)-\tilde{g} \nabla_{0} u(\tilde{q})\right| \leq|g-\tilde{g}|\left|\nabla_{0} u(q)\right|+\tilde{g}\left|\nabla_{0} u(q)-\tilde{g} \nabla_{0} u(\tilde{q})\right| .
$$

By (4.1), and the assumptions on $q$ and $\tilde{q}$,

$$
|g-\tilde{g}|\left|\nabla_{0} u(q)\right| \leq \delta g\left|\nabla_{0} u(q)\right| \leq C_{1} \delta .
$$

First by choosing $\beta<\frac{1}{2}$, then by (4.1) and (4.2), we have

$$
g\left|\nabla_{0} u(q)-\tilde{g} \nabla_{0} u(\tilde{q})\right| \leq C_{2} C_{1} \frac{1}{\frac{1}{2}-\beta} \frac{|q-\tilde{q}|}{d_{q \tilde{q}}}\left(\left|\log \frac{d_{q, \tilde{q}}}{|q-\tilde{q}|}\right|+1\right) .
$$

Since $\frac{|q-\tilde{q}|}{d_{q \tilde{q}}} \leq \frac{\beta}{\frac{1}{2}-\beta}$ and that $t \log t \rightarrow 0$ as $t \rightarrow 0$, we can choose $\beta$ sufficiently small such that

$$
\frac{|q-\tilde{q}|}{d_{q \tilde{q}}}\left(\left|\log \frac{d_{q, \tilde{q}}}{|q-\tilde{q}|}\right|+1\right) \leq \epsilon .
$$

By (4.3)-(4.5), we have

$$
\left|g \nabla_{0} u(q)-\tilde{g} \nabla_{0} u(\tilde{q})\right| \leq C_{1} \beta+C_{1} C_{2}(1+\beta) \frac{\epsilon}{\frac{1}{2}-\beta} .
$$

The lemma follows by choosing $\beta<\epsilon$ and sufficiently small.

Suppose $h: \mathbb{H}^{2} \rightarrow N$ is a proper harmonic map such that when viewed as a map from $\overline{\mathbb{D}^{2}}$ to $\overline{\mathbb{D}^{2}}$ is $C^{1}$ up to the boundary. Using the upper halfspace model for the domain and target, $h$ can be expressed as $h(x, y)=$ $(f(x, y), g(x, y))$.

Lemma 4.2. Let $h$ be a proper harmonic map from $\mathbb{H}^{2}$ to $N$, which is $C^{1}$ up to the boundary. Let $p_{i} \rightarrow o=(0,0)$ in the domain such that $q_{i}=h\left(p_{i}\right)=$ $\left(f, g_{i}\right)$ for some $f$ with $g_{i} \rightarrow 0$. Suppose $h$ is not conformal at $(0,0)$ then $g_{i} u\left(q_{i}\right) \rightarrow 1$ as $i \rightarrow \infty$.

Proof. Let $V$ be the intersection of $B_{o}(r)$ and the upper half-space containing all $p_{i}$ in the domain, and let $U$ be the intersection of $B_{q}(r)$ and the upper 
half-space in the target containing all $q_{i}$, where $q=(f, 0)$. Since $h$ is $C^{1}$ up to the boundary, there is a constant $C_{3} \geq 1$ such that for all $p, p^{\prime} \in \bar{V}$

$$
\left|h(p)-h\left(p^{\prime}\right)\right| \leq C_{3}\left|p-p^{\prime}\right| .
$$

In particular, if $p_{i}=\left(x_{i}, y_{i}\right)$, since $h\left(x_{i}, 0\right)=\left(f_{i}, 0\right)$ we have

$$
g_{i} \leq C_{3} y_{i}
$$

Given $\epsilon>0$ let $0<\beta<\frac{1}{2}$ be the constant in Lemma 4.1 for the neighborhood $U$. Let $\epsilon_{i}=\frac{\beta g_{i}}{C_{3}}$, then $\epsilon_{i} \leq \beta y_{i}<\frac{1}{2} y_{i}$ because of (4.7). Therefore $B_{p_{i}}\left(\epsilon_{i}\right)$ lies in the interior of the upper half-space. Without loss of generality, since $\epsilon_{i} \rightarrow 0$ as $i \rightarrow \infty$, we may assume that $B_{p_{i}}\left(\epsilon_{i}\right)$ lies inside $V$ for all $i$. By the argument of [L-T 2], there is a sequence $\left\{\tilde{p}_{i}\right\}$ such that $\left|p_{i}-\tilde{p}_{i}\right| \leq \epsilon_{i}$ and

$$
\epsilon_{i} \Delta_{0} f\left(\tilde{p}_{i}\right) \rightarrow 0
$$

as $i \rightarrow \infty$. If we write $\tilde{q}_{i}=h\left(\tilde{p}_{i}\right)=\left(\tilde{f}_{i}, \tilde{g}_{i}\right)$, then by (4.6), we have

$$
\begin{aligned}
\left|q_{i}-\tilde{q}_{i}\right| & =\left|h\left(p_{i}\right)-h\left(\tilde{p}_{i}\right)\right| \leq C_{1}\left|p_{i}-\tilde{p}_{i}\right| \\
& \leq C_{1} \epsilon_{i}=\beta g_{i} .
\end{aligned}
$$

Since $h$ is harmonic, using (3.5) in the rectangular coordinates $(x, y)$ for the domain and $(f, g)$ for the target, we have

$$
g \Delta_{0} f=g u_{1}\left(\left|\nabla_{0} g\right|^{2}-\left|\nabla_{0} f\right|^{2}\right)-2\left(g u_{2}-1\right)\left\langle\nabla_{0} f, \nabla_{0} g\right\rangle
$$

and

$$
g \Delta_{0} g=-\left(g u_{2}-1\right)\left(\left|\nabla_{0} g\right|^{2}-\left|\nabla_{0} f\right|^{2}\right)-2 g u_{1}\left\langle\nabla_{0} f, \nabla_{0} g\right\rangle,
$$

where $u_{1}=\frac{\partial u}{\partial f} \circ h$ and $u_{2}=\frac{\partial u}{\partial g} \circ h$. Therefore, (4.8), (4.9) and the definition of $\epsilon_{i}$ implies that

$$
\tilde{g}_{i} u_{1}\left(\tilde{q}_{i}\right)\left(\left|\nabla_{0} f\right|^{2}\left(\tilde{p}_{i}\right)-\left|\nabla_{0} g\right|^{2}\left(\tilde{p}_{i}\right)\right)+2\left(\tilde{q}_{i} u_{2}\left(\tilde{q}_{i}\right)-1\right)\left\langle\nabla_{0} f\left(\tilde{p}_{i}\right), \nabla_{0} g\left(\tilde{p}_{i}\right)\right\rangle \rightarrow 0
$$

as $i \rightarrow \infty$. By (4.9) and Lemma 4.1,

$$
\left|\tilde{g}_{i} u_{k}\left(\tilde{q}_{i}\right)-g_{i} u_{k}\left(q_{i}\right)\right| \leq \epsilon
$$

for all $i$ and for $k=1,2$. Since $h$ is $C^{1}$ up to the boundary, because of (4.1), (4.10), and (4.11), we see that

$$
\begin{aligned}
& \limsup _{i \rightarrow \infty} \mid g_{i} u_{1}\left(q_{i}\right)\left(\left|\nabla_{0} f\right|^{2}\left(p_{i}\right)-\left|\nabla_{0} g\right|^{2}\left(p_{i}\right)\right) \\
&+2\left(q_{i} u_{2}\left(q_{i}\right)-1\right)\left\langle\nabla_{0} f\left(p_{i}\right), \nabla_{0} g\left(p_{i}\right)\right\rangle \mid \\
& \leq 4 \epsilon C_{3}^{2} .
\end{aligned}
$$

Letting $\epsilon \rightarrow 0$, this implies that

$$
g_{i} u_{1}\left(q_{i}\right)\left(\left|\nabla_{0} f\right|^{2}\left(p_{i}\right)-\left|\nabla_{0} g\right|^{2}\left(p_{i}\right)\right)+2\left(q_{i} u_{2}\left(q_{i}\right)-1\right)\left\langle\nabla_{0} f\left(p_{i}\right), \nabla_{0} g\left(p_{i}\right)\right\rangle \rightarrow 0,
$$

as $i \rightarrow \infty$. Similarly, one can also prove that

$$
\left(g_{i} u_{2}\left(q_{i}\right)-1\right)\left(\left|\nabla_{0} f\right|^{2}\left(p_{i}\right)-\left|\nabla_{0} g\right|^{2}\left(p_{i}\right)\right)-2 q_{i} u_{1}\left(q_{i}\right)\left\langle\nabla_{0} f\left(p_{i}\right), \nabla_{0} g\left(p_{i}\right)\right\rangle \rightarrow 0
$$


as $i \rightarrow \infty$. The estimate $(4.1)$ asserts that

$$
\left(\left(g_{i} u_{2}\left(q_{i}\right)-1\right)^{2}+\left(g_{i} u_{1}\left(q_{i}\right)\right)^{2}\right)\left(\left|\nabla_{0} f\right|^{2}\left(p_{i}\right)-\left|\nabla_{0} g\right|^{2}\left(p_{i}\right)\right) \rightarrow 0,
$$

and

$$
\left(\left(g_{i} u_{2}\left(q_{i}\right)-1\right)^{2}+\left(g_{i} u_{1}\left(q_{i}\right)\right)^{2}\right)\left\langle\nabla_{0} f\left(p_{i}\right), \nabla_{0} g\left(p_{i}\right)\right\rangle \rightarrow 0
$$

as $i \rightarrow \infty$. If $h$ is not conformal at $(0,0)$ then

$$
g_{i} u_{2}\left(q_{i}\right)-1 \rightarrow 0
$$

as $i \rightarrow \infty$, and the proof of the lemma is completed.

Lemma 4.3. Let $h$ be a proper harmonic map from $\mathbb{H}^{2}$ onto $N$ which is $C^{1}$ up to the boundary when viewed as a map from $\overline{\mathbb{D}^{2}}$ to $\overline{\mathbb{D}^{2}}$. Suppose that the boundary map has nowhere vanishing energy density then $h$ is conformal at every point of the boundary.

Proof. Let us first prove that $h$ must be conformal at some boundary point. Assuming the contrary, since the boundary map has nowhere vanishing energy density $h$ must be surjective. Let $(f, 0)$ be a boundary point in the target. Consider any sequence $g_{i} \rightarrow 0$, and let $q_{i}=\left(f, g_{i}\right)$. Since $h$ is onto, there exists $p_{i}$ in the domain so that $h\left(p_{i}\right)=q_{i}$. Passing to a subsequece, we may assume without loss of generality that $p_{i} \rightarrow(0,0)$. Since $h$ is not conformal at $(0,0)$, Lemma 4.2 asserts that

$$
g_{i} u_{2}\left(q_{i}\right)-1 \rightarrow 0
$$

as $i \rightarrow \infty$. Since $\left\{g_{i}\right\}$ is an arbitrary sequence we conclude that $g u(q)-1 \rightarrow 0$ as $g \rightarrow 0$, where $q=(f, g)$. However this implies that $u$ is unbounded, which is a contradiction. Hence $h$ must be conformal at some point at the boundary. Since $h$ is $C^{1}$ up to the boundary, the set of boundary points at which $h$ is conformal is closed. Using the upper half-space model for the domain and the target. Suppose $h$ is conformal at $(0,0)$, then $g_{y}(0,0) \neq 0$ because $f_{x}(0,0) \neq 0$ and $g_{x}(0,0)=0$. Hence $g_{y} \neq 0$ near $(0,0)$. However, at a boundary point $(x, 0)$ so that $g_{y} \neq 0, h$ is a local $C^{1}$ diffeomorphism. Therefore if $h(x, 0)=(f, 0)$, then for any $g_{i} \rightarrow 0$, and if $q_{i}=\left(f, g_{i}\right)$ one can find a sequence $p_{i} \rightarrow(x, 0)$ such that $h\left(p_{i}\right)=q_{i}$. If $h$ is not conformal at $(x, 0)$, then by Lemma 4.2, we have $g_{i} u_{2}\left(f, g_{i}\right) \rightarrow 1$ as $i \rightarrow \infty$. Since $g_{i} \rightarrow 0$ is an arbitrary sequence, so $g u_{2}(f, g)-1 \rightarrow 0$ as $g \rightarrow 0$. This is impossible since $u$ is bounded. Therefore $h$ is conformal in a neighborhood of $(0,0)$ on the boundary. Hence the boundary points at which $h$ is conformal is also open. Since $h$ is conformal at some boundary point, so $h$ must be conformal everywhere at the boundary.

Theorem 4.4. Let $N$ be a hyperbolic Hadamard surfaces. Suppose $h_{1}$ and $h_{2}$ are two proper harmonic maps from $\mathbb{H}^{2} \rightarrow N$ such that when viewed as maps from $\overline{\mathbb{D}}^{2}$ to $\overline{\mathbb{D}}^{2}$ they are $C^{1}$. If the maps $h_{1}$ and $h_{2}$ when restricted to the boundary are given by the same boundary map $\phi: \mathbb{S}^{1} \rightarrow \mathbb{S}^{1}$ which has nowhere vanishing energy density, then $h_{1} \equiv h_{2}$.

Proof. By Lemma 4.3, both $h_{1}$ and $h_{2}$ are conformal on the boundary of $\overline{\mathbb{D}}^{2}$. Following the proof of Theorem 1.1 in [L-T 2] we conclude that the distance between the two maps with respect to the hyperbolic distance satisfies 
$d_{\mathbb{H}^{2}}\left(h_{1}(p), h_{2}(p)\right) \rightarrow 0$ as $p \rightarrow \infty$. The fact that $N$ is quasi-isometric to $\mathbb{H}^{2}$ implies that $d_{N}\left(h_{1}(p), h_{2}(p)\right) \rightarrow 0$ as $p \rightarrow \infty$. Moreover the assumption that $N$ has non-positive curvature implies that $d_{N}\left(h_{1}(p), h_{2}(p)\right)$ is subharmonic. The maximum principle asserts that $d_{N}\left(h_{1}, h_{2}\right)=0$ and hence $h_{1} \equiv h_{2}$.

\section{REFERENCES}

[A] K. Akutagawa, Harmonic diffeomorphisms of the hyperbolic plane, Trans. Amer. Math. Soc. (to appear).

[C-T] H. I. Choi and A. Treibergs, Gauss map of spacelike constant mean curvature hypersurfaces of Minkowski space, J. Differential Geom. 32 (1990), 775-817.

[C-Y] S. Y. Cheng and S. T. Yau, Differential equations on Riemannian manifolds and their geometric applications, Comm. Pure Appl. Math. 28 (1975), 333-354.

[G-T] D. Gilbarg and N. S. Trudinger, Elliptic partial differential equations of second order, Springer-Verlag, Berlin-Heidelberg-New York, 1977.

[L-T 1] P. Li and L. F. Tam, The heat equation and harmonic maps of complete manifolds, Invent. Math. 105 (1991), 1-46.

[L-T 2] P. Li and L. F. Tam, Uniqueness and regularity of proper harmonic maps, Ann. of Math. 136 (1992), 169-203.

[L-T 3] _ Uniqueness and regularity of proper harmonic maps II, Indiana Univ. Math. J. 42 (1993), 591-635.

[S] R. Schoen, The role of harmonic mappings in rigidity and deformation problems, Complex Geometry, Lecture Notes in Pure and Appl. Math., 143, Dekker, New York, 1993, pp. 179-200.

[W] T. Wan, Constant mean curvature surface, harmonic maps and universal Teichmüller space, J. Differential Geom. 35 (1992), 643-657.

[Y] S. T. Yau, A generalized Schwarz lemma for Kähler manifolds, Amer. J. Math. 100 (1978), 197-203.

Department of Mathematics, University of California, Irvine, California 92717-3875

E-mail address: pli@math.uci.edu

E-mail address: Itam@math.uci.edu

Department of Mathematics, Stanford University, Stanford, California 94305-2125

E-mail address: jwang@math.stanford.edu 